\title{
High speed indentation measures by FV, QI and QNM introduce a new understanding of bionanomechanical experiments
}

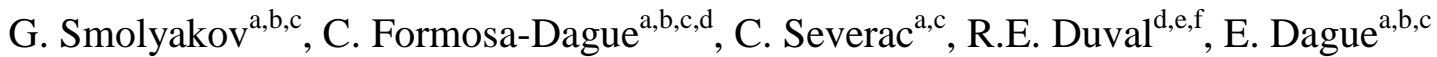

${ }^{a} I T A V$, Université de Toulouse, CNRS, UPS, France

${ }^{b}$ LAAS, Université de Toulouse, CNRS, UPS, France

${ }^{c}$ Université de Toulouse, Toulouse, France

${ }^{d}$ CNRS, SRSMC (Structure et Réactivité des Systèmes Moléculaires Complexes), UMR 7565, Nancy, France

${ }^{e}$ Université de Lorraine, SRSMC, UMR 7565, Faculté de Pharmacie, Nancy, France

${ }^{f}$ ABC PlatformR, Nancy, France

\section{ABSTRACT}

Structural and mechanical mapping at the nanoscale by novel high-speed multiparametric Quantitative Imaging (QI) and PeakForce Quantitative Nanomechanical Mapping (PF-QNM) AFM modes was compared to the classical Force Volume (FV) mapping for the case of living Pseudomonas aeruginosa bacterial cells. QI and PF-QNM modes give results consistent with FV for the whole cells in terms of morphology and elastic modulus, while providing higher resolution and shorter acquisition time. As an important complement, the influence of scanning parameters on elastic modulus values was explored for small $0.2^{2} \mu \mathrm{m}^{2}$ central area on top of cells. The modulus decreases with the indentation depth due to the effect of the hard cell wall, while it increases versus tip oscillation frequency, displaying viscoelastic behaviour of the living bacterial cells. The ability of different AFM modes to follow correctly the bacteria viscoelastic behaviour at high oscillation frequency was tested.

\section{Keywords:}

Atomic force microscopy

Force volume

Quantitative imaging

PeakForce quantitative nanomechanical mapping

Living bacterial cells

Elastic modulus 


\section{Introduction}

Atomic force microscopy (Binnig et al., 1982; Binnig and Quate, 1986) has become a powerful tool for structural and mechanical mapping of biological samples at the nanoscale (Muller et al., 2009; Dufrêne et al., 2008; Cross et al., 2007; Liu and Wang, 2010; Dorobantu and Gray, 2010; Formosa et al., 2012a; Formosa et al., 2012b; Yao et al., 2002; Aguayo et al., 2015). Since the development of the Force Volume (FV) mode in 1994 (Radmacher et al., 1994), it became possible to obtain force curves related to the interaction between the AFM tip and the sample surface. These curves give access to the surface topography and mechanical properties simultaneously. In particular, sample deformation and elastic modulus can be extracted, as shown in Fig. 1a. For that, the approach force curve is fitted with an appropriate geometric model. On the other hand, the retract curve may be used in order to calculate the adhesion force. The latter corresponds to the minimum force and may have nonspecific or specific nature.

In the FV mode, the tip approaches the surface until a certain force, called peak force, is reached. Then the tip is retracted and moves to the next position (Fig. 1b). Recently a new solution has been proposed by Bruker Corporation: a high-speed multiparametric mode called PeakForce Quantitative Nanomechanical Mapping (PF-QNM) (Hu et al., 2011; Alsteens et al., 2012; Dufrêne et al., 2013; Martín-Rodríguez et al., 2014). In this mode the tip pathway is modified, allowing faster scans. The height and mechanical properties images are provided in real time, while the registration of force curves is an additional option. In its turn, JPK Instruments has also developed a multiparametric mode, called Quantitative Imaging (QI) (Chopinet et al., 2013a; Chopinet et al., 2013b). Analysis of the saved force curves in this mode gives access to the sample height and mechanical behaviour. A comparison of FV, PF-QNM and QI modes is given in Fig. 1c. The PF-QNM mode works at the highest oscillation frequency and can adopt a number of discrete values from $0.25 \mathrm{kHz}$ to $8 \mathrm{kHz}$. For biological research a relatively low frequency of $0.25 \mathrm{kHz}$ or $0.5 \mathrm{kHz}$ is usually applied. In the FV and QI modes the frequency range is about $0.5 \div 100 \mathrm{~Hz}$. On the other hand, the minimum resolution in FV and QI is much lower $\left(8^{2} \mathrm{px}^{2}\right)$ compared to PF-QNM $\left(128^{2} \mathrm{px}^{2}\right)$, thus allowing very fast scans, if detailed surface map is not required. In its turn, PF-QNM is the only mode in which a good statistics for every pixel in terms of 
number of force curves is possible. Resulting value for a pixel represents an average from several force curves. This is very useful for nanomechanical or nonspecific adhesion investigations, while it has no sense for specific adhesion measurements, as the average value will depend on the probability of specific adhesion events. Thus in order to measure the specific adhesion value correctly, the PF-QNM parameters should be adapted in the way to have only one force curve per pixel. On the other hand, very high speed of PF-QNM may prevent a specific bond establishment, as the latter takes some time. As for the FV and QI, in the latter modes only one force curve contributes to each pixel.

Pseudomonas aeruginosa $(\mathrm{Pa})$ is an invasive, Gram-negative pathogen that causes a wide range of severe infections including bacteraemia, pneumonia, meningitis, urinary tract and wound infections (Hauser and Ozer, 2011). PaR3 (kindly provided by Pr. R.E. Duval from ABC Platform, Nancy, France) has been taken as an object for the present study, being a clinical multiresistant strain (antibiogram is shown in Supplementary data 1).

The main aim of the present work was to apply Nanowizard III (JPK Instruments) and Bioscope Catalyst (Bruker Corporation) instruments for elucidating the nanomechanical behavior of living bacterial cells when indented at high frequency with AFM. The study was limited to these two instruments, although other manufactures propose as well the solutions for probing the bionanomechanical properties. We used the classical FV mode and the novel high-speed QI and PF-QNM modes. The work has been performed using the following strategy: first, the whole bacteria were explored by the three AFM modes in order to compare the cell morphology, as well as the elastic modulus variation across the cells; second, small $0.2^{2} \mu \mathrm{m}^{2}$ zones in the centre of the cells were scanned in order to see a dependence of the elastic modulus from major scanning parameters: applied force and oscillation frequency; third, the possibility of very fast FV scans (classically considered as a slow mode) was explored with both JPK and Bruker instruments, using bacteria as well as standard PDMS samples.

\section{Materials and methods}

\subsection{Bacterial sample preparation}


PaR3 were stocked at $-80{ }^{\circ} \mathrm{C}$, revivified on Mueller Hinton agar (Difco, 225250-500 g) and grown in $5 \mathrm{~mL}$ of Mueller Hinton broth (Difco, $275730-500 \mathrm{~g}$ ) for $24 \mathrm{~h}$ at $35^{\circ} \mathrm{C}$ under static conditions. Then $10 \mu \mathrm{L}$ of the cells suspension were introduced to $5 \mathrm{~mL}$ of Mueller Hinton broth for $24 \mathrm{~h}$ at $35^{\circ} \mathrm{C}$ under static conditions. The same procedure was repeated one more time for a good standardization of bacterial cell cultures. PaR3 cells were then immobilized on polyethylenimine (PEI, Fluka P3124-100 mL) coated glass slides as described previously (El Kirat et al., 2005). Briefly, freshly oxygen plasma activated glass slides were covered by a $0.2 \%$ PEI solution in deionized water and left for incubation overnight. Then glass slides were rinsed with 10 $\mathrm{mL}$ of Milli-Q water and nitrogen dried. Bacterial cultures were harvested by centrifugation, washed two times in PBS, and resuspended to a concentration of $\sim 10^{8}$ cells $/ \mathrm{mL}$ in phosphate buffered-saline $1 \mathrm{X}(10 \mathrm{mM})$ (PBS, Sigma, P2194-10PAK). $1 \mathrm{~mL}$ of the suspension was deposited on the PEI coated glass slide and allowed to stand for 20 min before rinsing with PBS 1X.

\subsection{Atomic force microscopy}

Two instruments were used for the present study: Nanowizard III (JPK Instruments) and Bioscope Catalyst (Bruker Corporation). As for the scanning conditions for both bacterial and PDMS samples, ramp size was set to $500 \mathrm{~nm}$, peak force to $1 \mathrm{nN}$, except for the experiment with increased penetration depth close to the cell diameter, for which the peak force was increased up to $8 \mathrm{nN}$. The oscillation frequency was set to $6 \mathrm{~Hz}$ for $\mathrm{FV}, 40 \mathrm{~Hz}$ for QI and $250 \mathrm{~Hz}$ for PF-QNM (if not specified otherwise). The images resolutions were $32^{2} \mathrm{px}^{2}$ for FV and $128^{2} \mathrm{px}^{2}$ for QI and PF-QNM modes. Measurements were done in a physiological PBS $1 \mathrm{X}$ buffer, using MLCT-C cantilever (if not specified otherwise) with a nominal spring constant of $10 \mathrm{mN} / \mathrm{m}$ (with an average value of $12.5 \mathrm{mN} / \mathrm{m}$ ). The thermal tuning method (Emerson Iv and Camesano, 2006) was used to calibrate the spring constant of the cantilever before each experiment.

The SPIP software was used for the analysis of the FV and QI results, with the force curves correction stage consisted in the noise reduction and the base line adjust. As for the PF-QNM, this mode provided the results in real time during the experiment.

\subsection{Elastic model}


Hertz elastic model for spherical contact between tip and surface (Hertz, 1882) was chosen for the analysis of the FV and QI data. MLCT (Bruker Corp.) cantilevers with a nominal tip radius of $20 \mathrm{~nm}$ were used for the present study. Cell maximum deformation at peak force was about $40 \mathrm{~nm}$ corresponding to an average deformation around $20 \mathrm{~nm}$ along the fitted part of the force curve. Although for the average deformation close to the tip nominal radius both Hertz and Sneddon (Sneddon, 1965) models are valid, we chose the Hertz one for the best fit of the small indentation (lower than the tip nominal radius) part of the force curve. The Hertz model links the sample deformation $d$ to the applied force $F$ via elastic modulus $E$ and tip radius $R$ values: $F=$ $4 / 3 * E /\left(1-v^{2}\right) * R^{1 / 2} * d^{3 / 2}$, with $v$ being sample Poisson ration, arbitrary taken as 0.5 .

The use of the MLCT-C cantilevers and $1 \mathrm{nN}$ as the force set point resulted in the maximum cantilever deflection around $80 \mathrm{~nm}$, that is about two times larger compared to the sample deformation. This allowed precise subtraction of cantilever deflection from the whole piezoelectric movement. On the other hand, the cantilever was highly sensitive allowing following in details the shape of force curves.

Instead of performing the tip radius calibration on a standard sample, which can damage or contaminate the tip, the same cantilever was used for different modes to account for a possible error in the tip radius value. As for the absolute modulus values, the latter have rather weak dependence from the tip radius $\left(E \sim R^{-1 / 2}\right)$, in addition these modulus values are relative by the AFM technique in principle.

As the PF-QNM provides the results in real time and the Hertz model is not embedded in the Bruker's online software, thus the Sneddon model was used for the modulus fit. The Sneddon model operates with the tip half-angle $\alpha$ (equal to $17.5^{\circ}$ for the MLCT-C cantilevers) as the geometric parameter of the tip: $F=$ $2 / \pi^{*} E /\left(1-v^{2}\right) * \tan \alpha * d^{2}$. As the sample deformation is increased in the PF-QNM compared to the QI or FV, the use of the Sneddon model for the PF-QNM measurements is indeed reasonable. The application of both Hertz and Sneddon models for the FV data showed that the difference between the modulus values by two models is as low as $10 \%$ (which shows again that both models could be applied). This difference in modulus by two models is much lower compared to the differences between AFM modes, which are discussed in this article.

So the bacterial cells mechanics is considered within the elastic approach in this work, without quantification of viscoelastic contribution, which may be important in real bacterial cells (Vadillo-Rodriguez 
et al., 2008; Vadillo-Rodriguez et al., 2009; Vadillo-Rodriguez and Dutcher, 2011). On the other hand, the cells are considered as homogeneous materials in order to apply the elastic models. In reality the mechanical response comes from different cellular components depending on the penetration depth.

\section{Results and discussion}

\subsection{Study of the whole PaR3 cells}

\subsubsection{Morphology}

The whole cells morphology was explored by FV, QI and PF-QNM modes, with a scan size set to $3.5^{2} \mu \mathrm{m}^{2}$ (Fig. 2). The bacteria diameter for a large majority of cells in a population varies between 350 and $650 \mathrm{~nm}$. This diameter is defined as the height of adherent cell. The diameter determined as the width of the cell is about two times larger. But the latter way of diameter estimation is wrong following the next two reasons. First, finite tip size causes overestimation of the cell width (geometric artefact). Second, the cells are adherent to the substrate, so their width is increased. The latter fact means as well that the bacteria height is underestimated. Thus, the PaR3 cells diameter from the AFM measurements is $350-650 \mathrm{~nm}$, being a bit underestimated compared to the free non deformed cells. According to the literature, the diameter of $P a$ cells is $0.5-1 \mu \mathrm{m}$ (Graham and Cady, 2014). In order to measure the cells diameter by AFM more properly, other technique of cells immobilization should be used instead of electrostatic one. For example, mechanical immobilization in pores of polycarbonate membranes (Dague et al., 2007) or holes of PDMS stamp (Dague et al., 2011; Formosa et al., 2015) could be used. However, the non-spherical form of PaR3 cells may provide problems for this latter mechanical immobilization. Coming back to our results, all three AFM modes give the same bacteria height. The height variations for different measurements are only related to variations in the cells morphology.

The repetitive measurements did not display significant changes on bacteria surface, thus the plastic deformation (that is remaining or irreversible deformation) does not play an important role.

\subsubsection{Elastic properties}


The elastic modulus maps of the PaR3 cells were also obtained for all three FV, QI and PF-QNM modes (Fig. 3). The modulus varies across the cell, increasing towards the center of the cell. This observation is rather interesting. First, it means that there is no substrate effect, otherwise the modulus would be higher towards the cell periphery, where the sample thickness is lower. Second, the bacterium cell does not contain hard organelles, which could cause modulus increase in the specific zone of the cell (Longo et al., 2012). So, this increase is probably related to geometric artefacts. At the point of maximum cell height the contact between the tip and the surface satisfies the most the condition of symmetric form (Hertz and Sneddon models). So the modulus estimation at this point is the most correct. On the contrary, the tip-surface contact becomes asymmetrical towards the cell edge, thus the modulus estimation following Hertz or Sneddon models becomes wrong. On the other hand, the elastic modulus which corresponds to the substrate is very high and cannot be measured correctly with such a flexible cantilever. The modulus increase towards the cells center was demonstrated before for Gram-negative Shewanella putrefaciens bacterial cells (Gaboriaud et al., 2008). In the same work a large increase of cells stiffness due to the substrate effect was observed, if the indentation depth exceeded around $60 \%$ of the cell thickness.

Fig. 3 shows as well the typical force curves for the three AFM modes recorded on PaR3 cells. The curves are displayed in two representations. First, the applied force is plotted against the piezoelectric element movement. The latter represents the sum of the tip-sample separation and cantilever deflection. If the deflection is subtracted, the curves in terms of force against separation are obtained, being shown as well. As one may see, the force curves obtained by QI mode contain rather intense noise, but the latter is easily removed during curves correction stages, and is not disturbing for the fit by a geometric model.

\subsection{Effect of the scanning conditions on the elastic modulus of PaR3 cells}

\subsubsection{Effect of the penetration depth}

The FV force curves obtained for small $0.2^{2} \mu \mathrm{m}^{2}$ zones in the central part of the bacteria were analysed to extract the modulus variation against the penetration depth. The FV curves were segmented into equal penetration length sections that were each fitted with the Hertz model. Two different applied forces were used to probe the elastic modulus at different depth. Three cells were analysed in every case. 
First, MLCT-C cantilevers with nominal spring constant of $10 \mathrm{mN} / \mathrm{m}$ were used to apply the maximum force of $1 \mathrm{nN}$. Such common maximum force yielded the deformation around $40 \mathrm{~nm}$. The resulting force curves were then broken into segments of $10 \mathrm{~nm}$ width, that were fitted using Hertz model (Fig. 4a). The elastic modulus decreases against penetration depth for the first $40 \mathrm{~nm}$ of indentation. This is a specific behaviour, which visualises the effect of hard cell wall, present in bacteria cells.

To reach deeper inside the cell, we used MLCT-D cantilever with the nominal spring constant of $30 \mathrm{mN} / \mathrm{m}$ (with an average value of $35 \mathrm{mN} / \mathrm{m}$ ). It was then possible to reach a maximum force of $8 \mathrm{nN}$ resulting in a penetration depth comparable to the bacterium diameter (Fig 4b). For these high indentations, the elastic modulus increases extremely due to the proximity of the hard substrate beneath the indented cell, or/and to incompressibility of cellular membrane and cytosol. The Hertz model is also used for the modulus estimation. This could seem incorrect, as the deformation is much higher compared to the tip radius. But this consideration has no sense, as the modulus in close proximity of the substrate becomes too high and cannot be measured with such a flexible cantilever. In addition the elastic response becomes extremely non linear, preventing correct application of Hertz model, which suggests the sample homogeneity. Thus the extreme modulus increase cannot be quantified in our test. Interestingly, for PDMS films (relatively soft material) the pronounced substrate effect takes place at deformation corresponding to $45 \%$ of sample thickness (Xu et al., 2011). As we see from our measurements, for living cells due to their flexibility in changing the shape under applied stress, the substrate effect combined with the effect of cellular material incompressibility happens at much later stage. Another interesting observation is that even totally compressed by the AFM tip, PaR3 cells preserve their viability at least at the timescale of the experiment, as the mechanical behaviour in the end of the scan is the same as in the beginning. Such a high resistance against mechanical compression have been shown already for Gram negative Salmonella typhimurium bacteria cells (Suo et al., 2009). Moreover, in the latter study the cells were punctured by an AFM tip when the latter passed through the membrane, as indicated by a specific peak on the approach part of the force curves. Nevertheless the cells stayed alive due to a selfrepairing of lipid bilayers and the peptidoglycan layer. In our case the membrane is not punctured, as no specific peak at approach force curve is present. In the same time, the authors also observed large modulus increase when the tip penetration was comparable to the cells diameter. 
The present determination of modulus variation against penetration depth allows defining the large deformation region with rather linear elastic response. The applied force should correspond to this deformation region, if the whole cell is supposed to be tested, with the cytoplasm deformation playing the main role.

\subsubsection{Effect of the tip oscillation frequency}

The FV mode is usually applied at rather low oscillation frequency $<10 \mathrm{~Hz}$. In this work the following question was asked: could the FV mode provide proper force curves with correct modulus values at much higher frequency? Thus the FV mapping was performed with both Bioscope Catalyst and Nanowizard III for frequencies ranging from $3 \mathrm{~Hz}$ to $78 \mathrm{~Hz}$ for small $0.2^{2} \mu \mathrm{m}^{2}$ zones, and the corresponding modulus values were extracted (Fig. 5a-b). All measurements were performed on three different cells for each of three different bacteria preparations to ensure statistically relevant data.

In Fig. 5a and Fig. 5b we compare the FV mode between the JPK Nanowizard III and the Bruker Bioscope Catalyst. In the JPK AFM case, the elastic modulus increases against oscillation frequency, and the dependence is typical for the viscoelastic behaviour (Fig. 5a) (Costa, 2003; Guilak, 2000; Huang et al., 2004).

In the case of the Bruker AFM, the FV measurements provide proper modulus vs. frequency dependence in the low frequency range, while in the range $13-19 \mathrm{~Hz}$ the elastic modulus begins to decrease, displaying an abnormal behaviour (Fig. 5b).

Figures $5 \mathrm{c}$ and $5 \mathrm{~d}$ present the comparison between both high-speed modes: the QI mode from the JPK AFM and the PF-QNM mode from the Bruker AFM. Again, three PaR3 cells coming from each of three different cultures were analyzed. The modulus vs. frequency dependence from the QI mode (Fig. 5c) displays the expected viscoelastic behaviour of $P a R 3$ cells. These results are consistent with those found in the FV mode. On the contrary, the modulus values obtained by the PF-QNM mode were very low and further decreased with the frequency (Fig. 5d). This tendency for the PF-QNM mode seems to be a continuation of the tendency already observed for the FV mode on the Bioscope Catalyst. The decrease in elastic modulus against frequency for biological sample has already been reported by Dokukin and Sokolov (2015). They attributed this tendency to the creep relaxation. The latter consists in the fact that cell membrane needs some time to establish a stable contact with the tip, so if the latter oscillates too quickly, the contact area is 
decreased. So, at constant force the stress and consequently the deformation are increased, and the calculated modulus decreases. One may suppose that if the scan area is very small and neighbour sites of tip-surface contacts are very close, thus the cell surface is not fully relaxed after previous deformation, hence preventing an establishment of the symmetric contact after small lateral displacement of the tip. As a consequence, the real contact area decreases resulting in an underestimated elastic modulus. This mechanism may explain the PF-QNM mechanical properties results for the small scanned area. In other words, in the latter case a small surface area is overcharged by very frequent tapping and the surface has not enough time to recover; as a consequence, the symmetric tip-surface contact is not established after tip lateral displacement, leading to the decrease of the contact area and a lower measured elastic modulus. As we have verified, this behaviour is conserved for the $0.5^{2} \mu \mathrm{m}^{2}$ central zones (nearly the maximum possible scanning size within a PaR3 cell). In the same time, the correct mapping of the whole bacteria by PF-QNM mode is possible (Fig. 3c).

On the other hand, the operating of FV on the Bruker's and JPK's instruments seems to be different, as for the JPK's AFM the creep relaxation does not take place at the frequency, at which this phenomenon probably takes place on Bruker's AFM.

\subsection{Force curves by FV mode at elevated frequency at Nanowizard III and Bioscope Catalyst.}

\subsubsection{On PaR3 cells}

To explore in more details the difference in modulus variation at high frequency by FV mode on JPK's and Bruker's instruments, the shape of individual force curves was considered (Fig. 6a-b). At the highest explored frequency of $78 \mathrm{~Hz}$ the force curves recorded with the Nanowizard III still preserve their shape, with well defined indentation region on both approach and retract parts of the curves (Fig. 6a). We were not able to obtain such proper curves at $78 \mathrm{~Hz}$ with the Bioscope Catalyst (Fig. 6b). Indeed, the approach curve still displays an indentation region, but this region becomes rather flat, thus the calculated elastic modulus is very low. As for the retract curve, any calculations of elastic modulus, adhesion or dissipation values would be impossible, as the curve has completely lost its proper shape.

\subsubsection{On PDMS standard sample}


To confirm a general character of the found FV mode features on JPK's and Bruker's instruments, a standard homogeneous PDMS sample was explored as well in the PBS medium (Fig. 6c-d). The PDMS is rather soft material (elastic modulus around several MPa depending on preparation), nevertheless it is too hard to be tested by MLCT-C cantilevers. Thus only cantilevers were tested in this experiment. The FV scan at 78 $\mathrm{Hz}$ on the Nanowizard III has provided again proper force curves with an indentation region available for the fitting process (Fig. 6c). It is interesting to note the presence of small adhesion, which is absent in the case of PaR3 cells. A non-zero adhesion on a hydrophobic PDMS sample means that although the AFM tip is hydrophilic, containing $\equiv \mathrm{SiOH}$ surface groups, but its hydrophilic character is less pronounced compared to the water. So in the aqueous medium the non modified AFM tip is effectively hydrophobic with respect to the PDMS.

As for the FV scan on the Bioscope Catalyst, the frequency $78 \mathrm{~Hz}$ does not allows to get any force curves. Thus the oscillation at $39 \mathrm{~Hz}$ was applied to collect force curves (Fig. 6d). The approach part at this frequency still conserves a proper form, while the retract part of force curves does not present any defined indentation region. In addition, the adhesion force is abnormally high, being non realistic for the contact between the hydrophobic PDMS surface and the polar surface of AFM tip.

\section{Conclusions}

The present work aimed to explore the nanomechanical behaviour of living bacteria at high indentation frequencies by applying different AFM modes. We used the novel high-speed multiparametric QI and PFQNM AFM modes, as well as the classical FV mapping. For small scan area $\left(<0.5^{2} \mu \mathrm{m}^{2}\right)$ within bacterial cells we found that: i) elastic models remain relevant for indentation frequencies below $20 \mathrm{~Hz}$; ii) above $20 \mathrm{~Hz}, \mathrm{FV}$ and QI from JPK display the expected increase of Young modulus versus frequency, iii) while FV and PFQNM from Bruker demonstrated a paradoxical behavior, showing decrease of the Young modulus that we attribute to creep relaxation.

All three FV, QI and PF-QNM modes provide consistent morphology and elastic modulus maps of the whole bacteria. Thus, one may benefit from lower acquisition time and higher resolution by using the QI or PF-QNM modes. 
In order to optimize the scanning conditions, an effect of penetration depth on the modulus values was studied using the FV mode. Too low penetration depth $(<20 \mathrm{~nm})$ leads to an increase of measured modulus, as the hard cell wall is tested in such a case. On the other hand, if the penetration depth becomes comparable to the cell diameter, the elastic modulus increases dramatically due to the hard substrate effect and incompressibility of cellular membrane and cytosol.

To establish the tip frequency range appropriate to obtain analyzable results, the elastic modulus behaviour versus the tip frequency was studied by FV, QI and PF-QNM modes for a small central area of the PaR3 cells. The JPK's FV and QI modes displayed the expected viscoelastic behaviour of the cells at different tip frequencies. In particular, the JPK's FV mode could be used at frequencies at least up to $79 \mathrm{~Hz}$, which ensures much lower acquisition time $(<1 \mathrm{~min})$ compared to the typical one for this mode $(5-10 \mathrm{~min})$. At high tip frequency Bruker's FV and PF-QNM display a paradoxical decrease of the Young modulus that could be attributed to creep relaxation. These findings are also supported by the shape of the force curves on $P a R 3$ cells, as well as on standard PDMS sample. The curves remain appropriate at high frequency by JPK's modes, preserving an indentation region for the fit. At the same time, PF-QNM is well suited to large scan area at the whole cell scale, thus avoiding possible sample overcharging.

\section{Acknowledgement}

This work was supported by the "Fondation pour la Recherche Médicale" (grant ING21040129094). E.D. is a researcher at Centre National de la Recherche Scientifique (CNRS).

\section{References}

Binnig, G., Rohrer, H., Gerber, C. \&Weibel, E., 1982. Tunneling through a controllable vacuum gap. Appl. Phys. Lett. 40, 178-180.

Binnig, G., Quate, C.F., 1986. Atomic force microscope. Phys. rev. lett. 56, 930-933.

Muller, D.J., Helenius, J., Alsteens, D., Dufrene, Y.F., 2009. Force probing surfaces of living cells to molecular resolution. Nat. Chem. Biol. 5, 383-390. 
Dufrene, Y.F., Muller, D. J., 2008. Atomic Force Microscopy as a multifunctional molecular toolbox in nanobiotechnology. Nat. Nanotechnol. 3, 261-269.

Cross, S.E., Jin, Y.-S., Rao, J., Gimzewski, J.K., 2007. Nanomechanical analysis of cells from cancer patients. Nat. Nanotechnol. 2, 780-783.

Liu, S., Wang, Y., 2010. Application of AFM in microbiology: a review. Scanning 32, 61-73.

Dorobantu, L.S., Gray, M. R., 2010. Application of atomic force microscopy in bacterial research. Scanning 32, 74-96.

Formosa, C., Grare, M., Duval, R.E., Dague, E., 2012a. Nanoscale effects of antibiotics on P. aeruginosa. Nanomed. Nanotechnol. Biol. Med. 8, 12-16.

Formosa, C., Grare, M., Jauvert, E., Coutable, A., Regnouf-de-Vains, J.B., Mourer, M., Duval, R.E., Dague, E., $2012 b$. Nanoscale analysis of the effects of antibiotics and CX1 on a Pseudomonas aeruginosa multidrug-resistant strain. Scientific Reports 2, 575-583.

Yao, X., Walter, J., Burke, S., Stewart, S., Jericho, M.H., Pink, D., Hunter, R., Beveridge, T.J., 2002. Atomic force microscopy and theoretical considerations of surface properties and turgor pressures of bacteria. Colloids and Surfaces B: Biointerfaces 23, $213-230$.

Aguayo, S., Donos, N., Spratt, D., Bozec, L., 2015. Single-bacterium nanomechanics in biomedicine: unravelling the dynamics of bacterial cells. Nanotechnology 26, 062001.

Radmacher, M., Cleveland, J.P., Fritz, M., Hansma, H.G., Hansma, P.K., 1994. Mapping interaction forces with the atomic force microscope. Biophysical Journal 66, 2159-2165.

Hu, Y., Hu, S., Su, C., Shi, J., Ma, J., 2011. US Patent: US2011/0167524 A1.

Alsteens, D., Dupres, V., Yunus, S., Latgé, J.-P., Heinisch, J.J., Dufrêne, Y.F., 2012. High-Resolution Imaging of Chemical and Biological Sites on Living Cells Using Peak Force Tapping Atomic Force Microscopy. Langmuir 28, $n^{\circ} 49,16738-16744$.

Dufrêne, Y.F., Martínez-Martín, D., Medalsy, I., Alsteens, D., Müller, D.J., 2013. Multiparametric imaging of biological systems by force-distance curve-based AFM. Nat. Methods 10, $n^{\circ}$ 9, 847-854.

Martín-Rodríguez, A.J., González-Orive, A., Hernández-Creus, A., Morales, A., Dorta-Guerra, R., Norte, M., Martín, V.S., Fernández, J.J., 2014. On the influence of the culture conditions in bacterial antifouling bioassays and biofilm properties: Shewanella algae, a case study. BMC Microbiology 14, 102-115.

Chopinet, L., Formosa, C., Rols, M.P., Duval, R.E., Dague E., 2013a. Imaging living cells surface and quantifying its properties at high resolution using AFM in QITM mode. Micron 48, 26-33. 
Chopinet, L., Roduit C., Rols, M.P., Dague E., 2013b. Destabilization induced by electropermeabilization analyzed by atomic force microscopy. Biochimica et Biophysica Acta 1828, 2223-2229.

Hauser, A.R., Ozer, E.A., 2011. Pseudomonas aeruginosa. Nat. rev. Microbiol. 9, Poster.

El Kirat, K., Burton, I., Dupres, V., Dufrene, Y.F., 2005. Sample preparation procedures for biological atomic force microscopy. J. Microsc. 218, 199-207.

Emerson Iv, R.J., Camesano, T.A., 2006. On the importance of precise calibration techniques for an atomic force microscope. Ultramicroscopy 106, 413-422.

Hertz, H., 1882. Über die berührung fester elastischer Körper (On the contact of rigid elastic solids). J. reine und angewandte Mathematik 92, 156-171.

Sneddon, I.N., 1965. The relation between load and penetration in the axisymmetric boussinesq problem for a punch of arbitrary profile. Int. J. Eng. Sci. 3, 47-57.

Vadillo-Rodriguez, V., Beveridge, T.J., Dutcher, J.R., 2008. Surface viscoelasticity of individual gram-negative bacterial cells measured using atomic force microscopy. J. Bacteriol. 190, 4225-4232.

Vadillo-Rodriguez, V., Schooling, S.R., Dutcher, J.R., 2009. In situ characterization of differences in the viscoelastic response of individual gram-negative and gram-positive bacterial cells. J. Bacteriol. 191, 5518-5525.

Vadillo-Rodriguez, T.J., Dutcher, J.R., 2011. Viscoelasticity of the bacterial cell envelope. Soft Matter 7, 4101-4110.

Graham M.V., Cady N.C., 2014. Nano and microscale topographies for the prevention of bacterial surface fouling. Coatings 4, 37-59.

Dague E, Gilbert Y, Verbelen C, Andre G, Alsteens D, Dufrêne Y.F., 2007. Towards a nanoscale view of fungal surfaces. Yeast 24 (4), 229-237.

Dague E., Jauvert E., Laplatine L., Viallet B., Thibault C., Ressier L., 2011. Assembly of live micro-organisms on microstructured PDMS stamps by convective/capillary deposition for AFM bio-experiments. Nanotechnology 22, 395102-395108.

Formosa, C., Pillet, F., Schiavone, M., Duval, R.E., Ressier, L., Dague, E., 2015. Generation of living cell arrays for atomic force microscopy studies. Nature Protocols 10, 199-204.

Longo, G., Rio, L.M., Roduit, C., Trampuz, A., Bizzini, A., Dietler, G., Kasas, S., 2012. Force volume and stiffness tomography investigation on the dynamics of stiff material under bacterial membranes. J. Mol. Recognit. 25, 278284.

Gaboriaud, F., Parcha, B.S., Gee, M.L., Holden, J.A., Strugnell, R.A., 2008. Spatially resolved force spectroscopy of bacterial surfaces using force-volume imaging. Colloids Surf B Biointerfaces 62(2), 206-213. 
Suo Z., Avci R., Deliorman M., Yang X., Pascual D.W., 2009. Bacteria survive multiple puncturings of their cell walls. Langmuir 25 (8), 4588-4594.

Xu, W., Chahine, N., Sulchek, T., 2011. Extreme hardening of PDMS thin films due to high compressive strain and confined thickness. Langmuir 27, 8470-8477.

Costa, K.D., 2003. Single-cell elastography: probing for disease with the atomic force microscope. Disease Markers 19, $139-154$.

Guilak, F., 2000. The deformation behavior and viscoelastic properties of chondrocytes in articular cartilage. Biorheology 37, 27-44.

Huang, H., Kamm, R.D., Lee, R.T., 2004. Cell mechanics and mechanotransduction: pathways, probes, and physiology. American Journal of Physiology—Cell Physiology 287, C1-C11.

Dokukin M., Sokolov I., 2015. High-resolution high-speed dynamic mechanical spectroscopy of cells and other soft materials with the help of atomic force microscopy. Scientific Reports 5, 12630. 
(a)

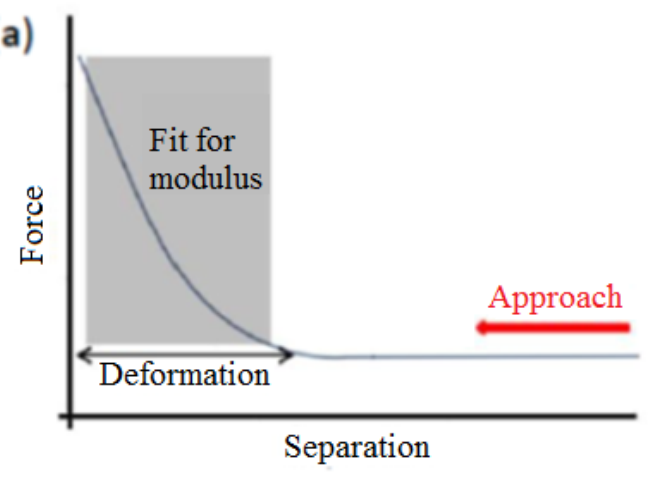

(b)

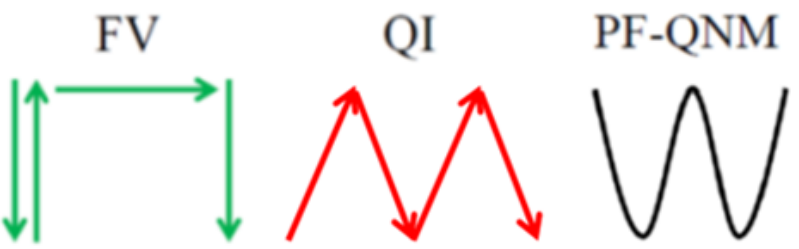

(c)

\begin{tabular}{c|c|c|c} 
& FV & QI & PF-QNM \\
\hline Frequency range $(\mathrm{Hz})$ & $\leq 80$ & $\leq 100$ & $250-500$ \\
Acquisition time $(\mathrm{s})$ & $\geq 0.8$ & $\geq 0.6$ & $\geq 60$ \\
Image resolution $\left(\mathrm{px}^{2}\right)$ & $8^{2} \div 64^{2}$ & $8^{2} \div 512^{2}$ & $128^{2} \div 512^{2}$ \\
Number of curves by pixel & 1 & 1 & $1-20$
\end{tabular}

Fig. 1. (a) Approach force curve used to calculate deformation and elastic modulus; (b) schematic representation of the tip pathway in FV, QI and PF-QNM modes; (c) comparative table for the three modes for the cases of Nanowizard III (JPK Instruments) and Bioscope Catalyst (Bruker Corporation) microscopes. Note that some systems can provide larger number of pixels but their usage depends rather on the time and patience of the experimenter, as well as on the time constrains imposed by living cells. 
(a)
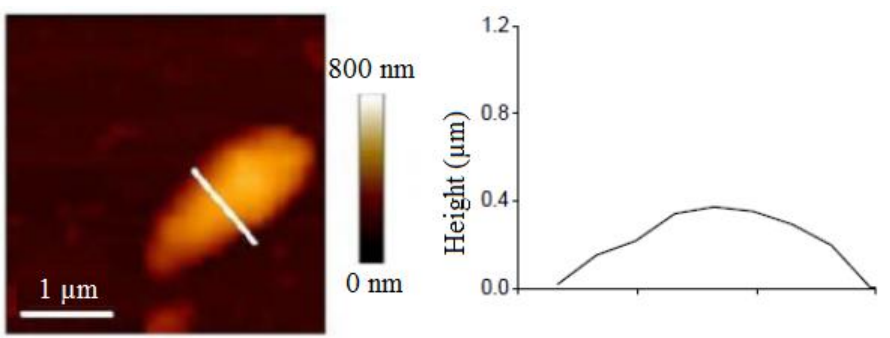

(b)
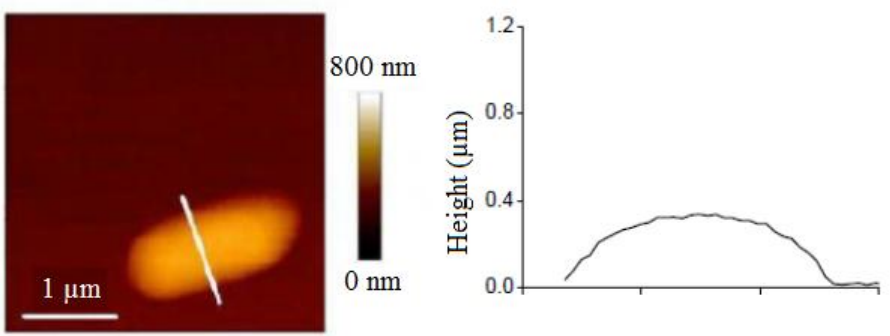

(c)
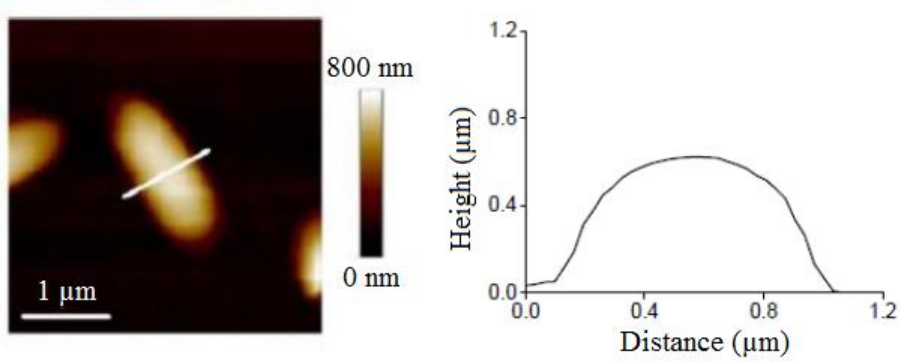

Fig. 2. Height images with corresponding profiles for $P a R 3$ in physiological buffer by $\mathrm{FV}\left(32^{2} \mathrm{px}^{2}\right.$ resolution$)$ (a), QI (128 ${ }^{2} \mathrm{px}^{2}$ resolution) (b) and PF-QNM $\left(128^{2} \mathrm{px}^{2}\right.$ resolution) (c) modes. The $\mathrm{x}$ and y scales are the same on the height profiles in order to see the real shape of the bacteria. 

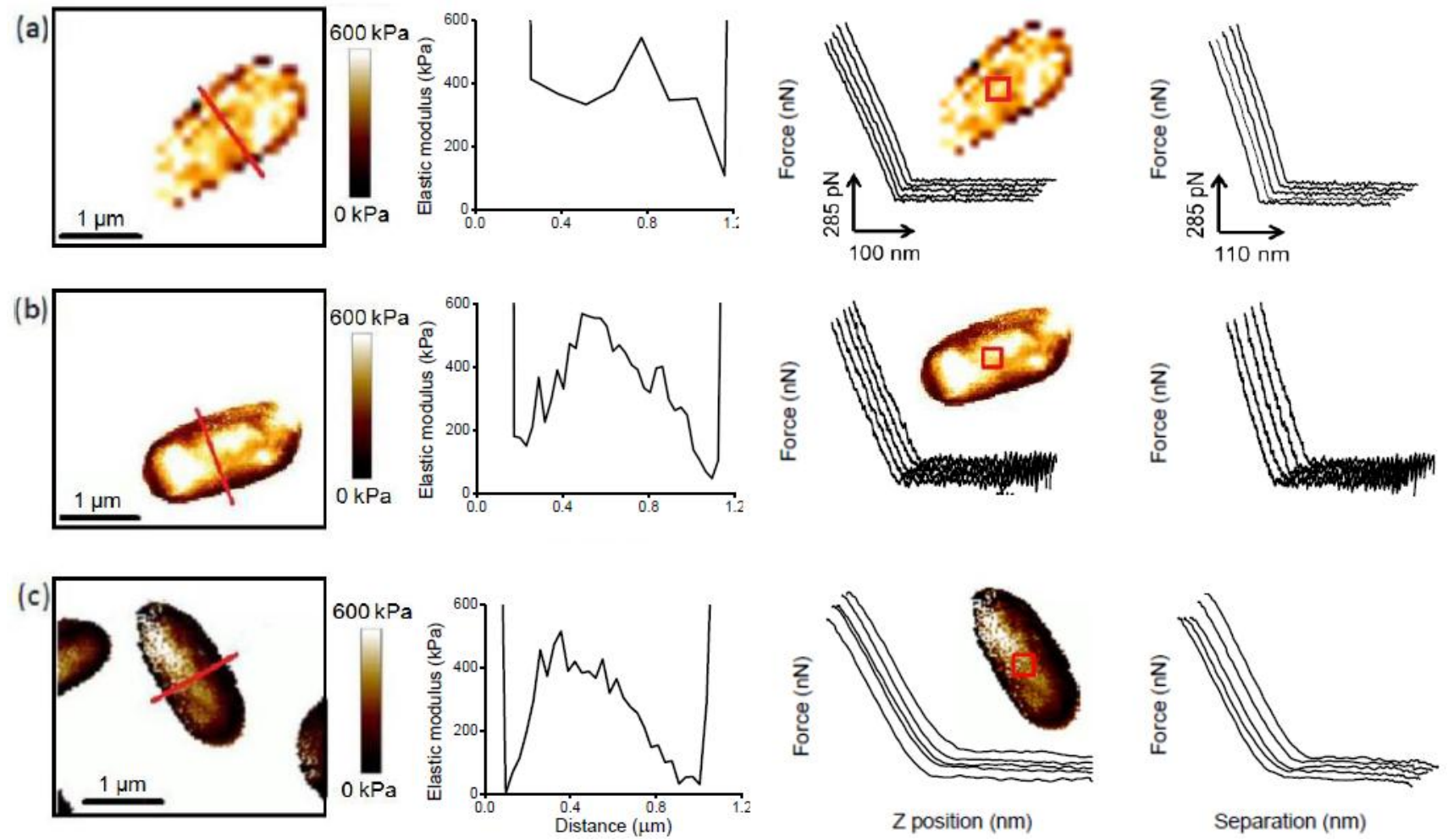

Z position (nm)

Separation $(\mathrm{nm})$

Fig. 3. Nanomechanical properties of PaR3 by FV (a), QI (b) and PF-QNM (c) modes. From left to right: elastic modulus map with resolution $32^{2} \mathrm{px}^{2}$ for $\mathrm{FV}$ and $128^{2} \mathrm{px}^{2}$ for QI and PF-QNM; corresponding profile, traced along the line at modulus map; five representative force curves in terms of $\mathrm{Z}$ position, collected in the region shown by square on the bacterium image; five representative force curves in terms of separation, with cantilever deflection being subtracted from $\mathrm{Z}$ position. 

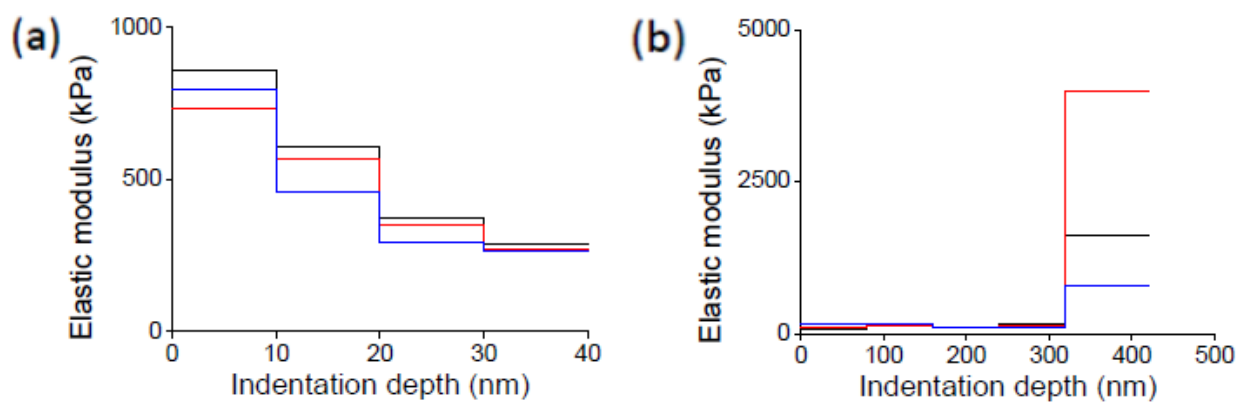

Fig. 4. Effect of the indentation depth on elastic modulus by FV mode for three different PaR3 cells represented by different colours: visualisation of the cell wall effect at small penetration depth (a); visualisation of the substrate effect and incompressibility of cellular membrane and cytosol at large penetration depth (b). 
(a)

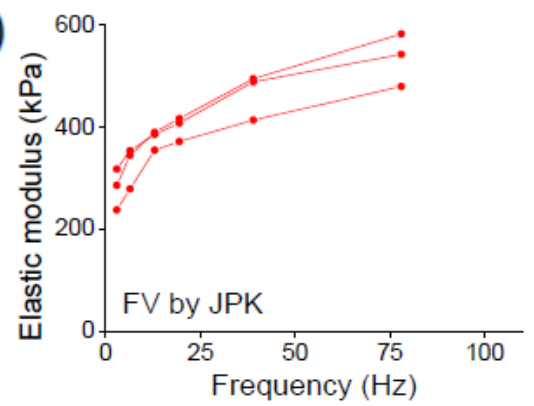

(c)

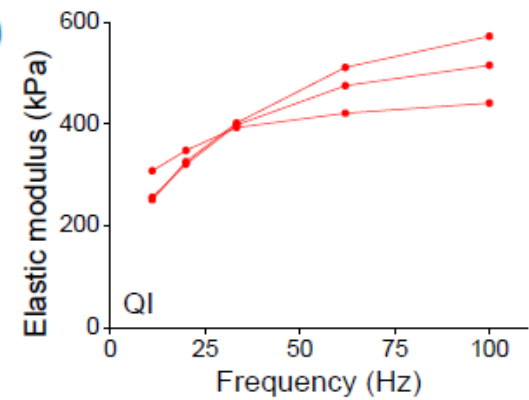

(b)

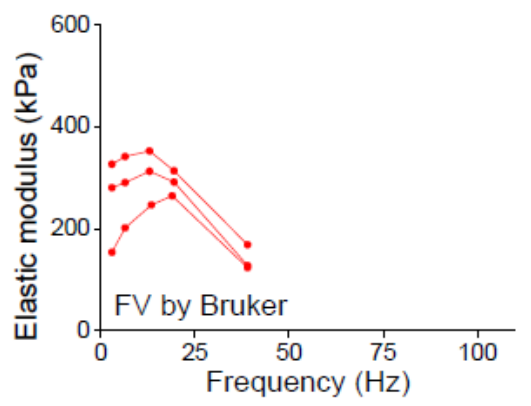

(d)

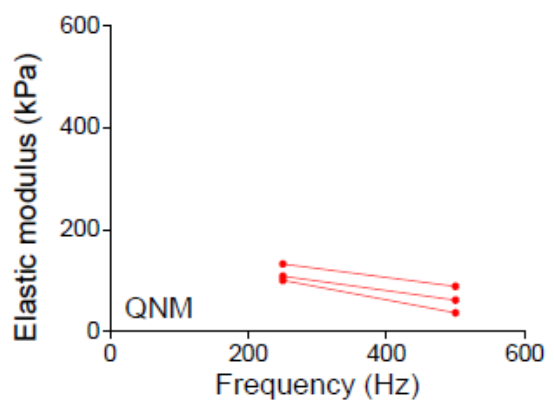

Fig. 5. Effect of the tip oscillation frequency on elastic modulus by different modes: FV on Nanowizard III (a); FV on Bioscope Catalyst (b); QI on Nanowizard III (c); PF-QNM on Bioscope Catalyst (d). 

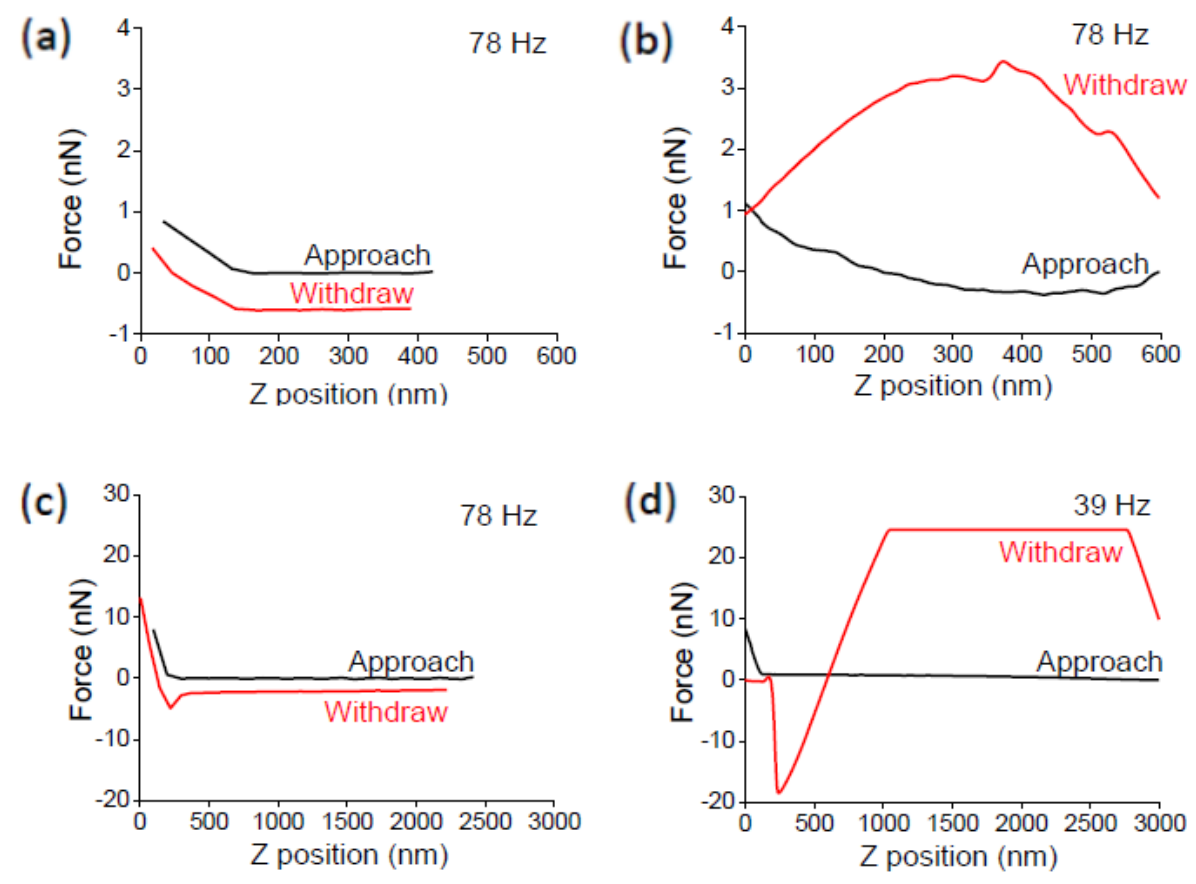

Fig. 6. Force curves at elevated frequency by FV mode. (a) PaR3 at Nanowizard III at $78 \mathrm{~Hz}$; (b) PaR3 at Bioscope Catalyst at $78 \mathrm{~Hz}$; (c) Standard PDMS at Nanowizard III at $78 \mathrm{~Hz}$; (d) Standard PDMS at Bioscope Catalyst at $39 \mathrm{~Hz}$ (as the frequency of $78 \mathrm{~Hz}$ does not allows to collect the curves in this case). 